\title{
Midkine, a cytokine that inhibits HIV infection by binding to the cell surface expressed nucleolin
}

\author{
Ara G Hovanessian ${ }^{1}$ \\ ${ }^{1}$ UPR 2228 CNRS, UFR Biomedicale-Université René Descartes, 45 rue des Saints Pères, 75270 Paris Cedex 6, France
}

The growth factor midkine (MK) is a cytokine that inhibits HIV infection in cell cultures in an autocrine and paracrine manner by blocking the attachment of HIV particles to permissive cells. MK mRNA is systematically expressed in adult peripheral blood lymphocytes from healthy donors, while its expression becomes markedly but transiently increased upon in vitro treatment of lymphocytes with IL-2 or IFN- $\gamma$ and activation of T lymphocytes by PHA or through the engagement of the CD28 antigen. The binding of MK to cells occurs specifically at a high and a low affinity binding site. This low affinity-binding site is the cell-surface expressed nucleolin, which is implicated in the mechanism of the initial attachment of HIV particles to cells. Accordingly, the nucleolin-binding HB-19 pseudopeptide has no effect on the MK binding to the high affinity binding site, whereas it prevents the binding of MK to the low affinity binding site, thus suggesting the low affinity receptor of MK is the cell-surface-expressed nucleolin. Confocal immunofluorescence laser microscopy revealed the colocalization of MK and the cell-surface-expressed nucleolin at distinct spots. The use of various deletion constructs of nucleolin then indicates that the extreme C-terminal end of nucleolin, containing repeats of the amino acid motif RGG, as the domain that binds MK. The specific binding of MK to the surface nucleolin is independent of heparan sulfate and chondroitin sulfate proteoglycans. After binding to cells, MK enters cells by an active process in which nucleolin and lipid rafts appear to be implicated. The potent and the distinct anti-HIV action of MK along with its enhanced expression in lymphocytes by various physiological stimuli, point out that MK is a cytokine that could be involved in HIV pathogenesis.

Cell Research (2006) 16:174-181. doi:10.1038/sj.cr.7310024; published online 13 February 2006

Keywords: HIV, Midkine, surface nucleolin, cytokine

\section{Cell surface expressed nucleolin serves as a receptor for HIV and MK}

HIV infects target cells by the capacity of its envelope glycoproteins gp120-gp41 to attach cells and induce the fusion of virus to cell membranes, a process which leads to virus entry. The HIV receptor complex essential for virus entry is composed of the CD4 molecule and at least one of the members of the chemokine receptor family (CXCR4 and CCR5). However, additional cell-surface components, such as heparan sulfate proteoglycans and nucleolin, appear to be implicated in the attachment of HIV particles. By using specific ligands, we have demonstrated that HIV

Correspondence: Ara G Hovanessian

Tel: +33-1-42864136

E-mail: ara.hovanessian@univ-paris5.fr attachment could be inhibited either by FGF-2 that uses heparan sulphate proteoglycans as low affinity receptors, or by the nucleolin binding pseudopeptide HB-19, that forms a stable complex with nucleolin $[1,2]$. The fact that HIV attachment is inhibited by two agents which do not compete, illustrates that virus attachment should be coordinated on one hand by heparan sulfate proteoglycans, and on the other hand by nucleolin. Consistent with the critical role of surface nucleolin in the initial steps of HIV infection, the HB-19 pseudopeptide and several physiological ligands of nucleolin (Midkine, Pleiotrophin, lactoferrin) inhibit HIV infection by blocking HIV particle attachment to CD4+ target cells [2-6].

Midkine (MK) and Pleiotrophin (PTN) are two related heparin-binding growth factors that are rich in basic amino acids and cysteines, and implicated in differentiation and development [7-8]. However, activated T cells from PBMC 
express MK but not PTN [3], the inhibition of HIV by MK and not PTN should be physiologically significant. The functional role of MK has been best demonstrated in the nervous system where MK enhances survival and neurite outgrowth of embryonic neurons and promotes neuronal differentiation [9]. MK is also known to promote plasminogen activator activity in aortic endothelial cells, leading to increased fibrinolysis. MK mRNA expression is increased in various human carcinomas and the MK protein has been reported to be required for the growth of the Wilm's tumour cells in vitro. Furthermore, MK has been shown to be accumulated in senile plaques of the brain of Alzheimer's disease patients. MK shares $45 \%$ sequence identity with PTN. Despite their striking structural homology and the conservation of their net positive charge, MK and PTN appear to have distinct receptors or receptor complexes. Four high affinity receptors for MK have been described in the literature: N-syndecans (chondroitin sulfate proteoglycans), Protein Tyrosine Phosphatase (PTP), Anaplastic Lymphoma Kinase (ALK), and Low-density-lipoprotein Receptor-related Protein (LRP). The precise mechanism by which these four receptors mediate signalling induced by MK is not yet determined. They might be differentially used by MK for specific biological activities [9]. In contrast to the high affinity receptors, the cell-surface expressed nucleolin is the only known low affinity receptor of MK [4]. Besides MK, surface nucleolin serves as a low affinity receptor for PTN [5] and lactoferrin [6].

\section{Expression of nucleolin at the cell surface}

Nucleolin is a RNA- and protein-binding multifunctional protein that has been previously described as being expressed mainly in the nucleolus $[10,11]$. Besides the nucleolus however, nucleolin is also expressed at the cell surface, where it acts as an integral membrane protein [12]. Indeed, incubation of intact cells with anti-nucleolin monoclonal antibody results in the clustering of membrane-expressed nucleolin. This clustering occurs at the external side of the plasma membrane as revealed by electron microscopy, thus confirming the surface expression of nucleolin.

The expression of the nucleolin at the cell surface was illustrated by using a specific antagonist of nucleolin, the HB-19 pseudopeptide that was designed initially as a potent inhibitor of HIV infection [13-16]. HB-19 presents pentavalently the tripeptide KPR in which the peptide bond between the $\mathrm{K}$ and $\mathrm{P}$ is reduced in order to increase the endopeptidase resistance of this pseudopeptide. HB-19 binds specifically various types of cells, and forms a stable and irreversible complex with the cell-surface expressed nucleolin. By this process, HB-19 prevents the interaction of various ligands with surface nucleolin, including HIV particles. By using various deletion constructs of nucleolin, we showed that the binding site of HB-19 is localized at the $\mathrm{C}$-terminal end of nucleolin: a domain of 60 amino acid residues known as the RGG domain that contains 9 repeats of the RGG motif [17].

The expression of surface nucleolin is tightly associated with cell proliferation and now has been shown in different types of cells [18]. Nucleolin mRNA is systematically detectable in peripheral blood lymphocytes of healthy individuals, and in vitro stimulation of proliferation results in an enhanced expression of nucleolin mRNA [3].

\section{MK is a cytokine that is expressed by activated $T$ lymphocytes}

MK mRNA is found to be expressed systematically in PBLs of healthy individuals, whereas pleiotrophin (PTN) and gamma interferon (IFN- $\gamma$ ) are not expressed. The level of MK mRNA expressed in vivo becomes undetectable $24 \mathrm{~h}$ following in vitro incubation of lymphocytes in the absence of any stimulation. However, MK expression is markedly induced by PHA or by the specific activation of T lymphocytes through the engagement of the CD28 antigen, using specific monoclonal antibodies. Cytokines, such as IL-2 or IFN- $\gamma$ are also effective for the induction of MK mRNA. Under similar experimental conditions, the PTN mRNA is not detectable at all, whereas IFN- $\gamma$ mRNA is induced only by PHA and IL-2 treatments. These observations indicate that MK is a cytokine induced by physiological stimuli.

The kinetics of MK mRNA expression indicated that there is a $24 \mathrm{~h}$ delay in MK expression compared to that of the well-known PHA-induced cytokine, IFN- $\gamma$. This result combined with the fact that IL-2 by itself is an inducer of MK expression, suggest that MK induction by PHA might be the consequence of IFN- $\gamma$ production. MK induction is transient, as the peak levels of induction at $2-4 \mathrm{~d}$ post-PHA activation of PBLs are followed by a marked decline. Therefore, MK expression is controlled by a regulatory mechanism that is commonly observed in the induction kinetics of cytokines and chemokines[19]. By confocal immunofluorescence microscopy a significant amount of endogenous MK was observed at the cell membrane of PBLs on the third day of PHA activation, compared to the corresponding cells before activation. The MK signal at the periphery of activated lymphocytes was markedly reduced on day 7 in accord with the transient induction of MK mRNA level during PHA activation of PBL. In contrast to the detection of surface MK, nucleolin was detectable on the surface of PBL before activation and its expression was increased and remained elevated in PHA-activated PBL. Interestingly, the surface MK 
staining colocalized with the nucleolin staining at the cell surface.

In order to avoid any interference on the HIV infection by MK produced during activation of PBLs, routinely HIV infections were carried out at 6 to $7 \mathrm{~d}$ post-PHA treatment when the MK mRNA levels were reduced.

\section{Inhibition of T-lymphocyte- and macrophage-tropic HIV-1 isolates in MK-Treated cells}

The inhibitory effect of MK on X4 and R5 HIV-1 entry and infection was first investigated in the experimental model of HeLa-CD4-LTR-lac Z cells expressing constitutively CXCR4 (HeLa P4 cells) or in addition expressing recombinant CCR5 (HeLa P4-C5 cells). HIV entry and replication in these cells result in Tat-mediated activation of the HIV LTR, leading to the expression of the lacZ gene. Consequently, the beta-galactosidase activity could be measured in cell extracts to monitor HIV entry into cells. In these well characterized HeLa experimental models, two commercially available preparations of human MK, either synthetic or recombinant were shown to inhibit the X4 HIV-1 LAI entry with an $\mathrm{IC}_{50}$ value of $25 \mathrm{nM}$ for MKB and $100 \mathrm{nM}$ for MKS. More than $90 \%$ inhibition occurred at $125 \mathrm{nM}$ of MKB and $250 \mathrm{nM}$ of MKS. The slightly lower activity of MKS compared to MKB was most probably due to differences that might exist between the chemically synthesized molecule and the recombinant protein generated by the bacterial system. MKS also inhibited entry of the R5 HIV-1 Ba-L and JR-CSF isolates, with $\mathrm{IC}_{50}$ value of 125 and $250 \mathrm{nM}$, respectively. In order to confirm the specificity of MK with respect to the HIV-envelope glycoprotein mediated viral-entry process, we investigated its inhibitory effect on infection by the HIV-1 pseudotype with envelope glycoproteins of vesicular stomatitis virus (VSV) or Moloney Murine Leukemia Virus (MoMLV). Infection by these pseudotyped HIV preparations was inhibited by AZT but not by the anti-CD4 mAb CB-T4, since the viral binding and entry is mediated by the pseudotyped viral envelope glycoproteins. Interestingly, MK as HB-19 exerted no significant effect on the pseudotyped HIV, thus pointing out that the inhibitory effect of these molecules is mediated on the entry process via the native HIV envelope glycoproteins.

To demonstrate the anti-HIV action of MK in primary CD + T lymphocytes, PHA-activated peripheral blood lymphocytes (PBLs) were treated with different doses of MKS before infection with the HIV-1 UG029A primary isolate. Without any treatment, a significant level of virus production was observed on the 6th day of infection with a first peak on day 8 and thereafter a decrease by day 12 . MK treatment caused a dramatic inhibition of virus infection and a significant delay on the onset of virus production. At $250 \mathrm{nM}$ of MK, low levels of virus production started to be detectable only on day 6 of infection, whereas at 500 $\mathrm{nM}$ of $\mathrm{MK}$, no virus production was detectable up to the 14 th $\mathrm{d}$ of infection.

\section{Mechanism of the anti-HIV action of MK}

MK is an efficient inhibitor of HIV infection when added to cells before virus, whereas when added few hours after virus entry then it exerts no significant effect. This points out that the anti-HIV action of MK is at an early phase in the HIV infectious cycle, as it is the case with the HB-19 pseudopeptide. The effect of MK on the attachment of HIV-1 LAI and Ba-L particles was then investigated in two types of cells: permissive HeLa P4-C5 cells that express CD4, CXCR4, and CCR5, and non-permissive HeLa cells that express only CXCR4. In order to avoid any viral entry and endocytosis, these experiments were carried out at $4^{\circ} \mathrm{C}$. Under such experimental conditions, most of the virus associated with cells was shown to be on the cell surface, since its recovery was reduced by more than $90 \%$ after treatment of cells with trypsin. Furthermore, consistent with our previous reports, the neutralizing $\mathrm{mAb}$ specific to the sequence of the V3 loop of HIV-1 LAI inhibited significantly the attachment of this virus in both CD4+ and CD4- cells, thus pointing out that the attachment process was indeed mediated by the HIV envelope glycoprotein gp120. In both CD4+ and CD4- HeLa cell lines studied, MK inhibited drastically in a dose-dependent manner the attachment of HIV-1 LAI and Ba-L to cells [3]. The observation that MK inhibited HIV-attachment to CD4-cells indicates that the mechanism of action of MK is independent of HIV interaction with CD4. In addition, these results point out that the mechanism of action of MK is at a very early step in the HIV entry process.

\section{MK could exert both autocrine and paracrine activity against HIV infection}

In order to illustrate that MK could function as a cytokine against HIV infection, we investigated if MK could exhibit an autocrine and paracrine activity. For this purpose, we established CD4+ (CEM) and CD4- (HeLa) cell lines expressing MK constitutively [3], and illustrate that: A) by an autocrine way CD4+ cells producing MK resist HIV infection; B) by a paracrine way CD4 -ve cells producing MK, when are cocultured with CD4 +ve cells, MK present in the culture medium binds to CD4+ bystander cells and protects them against HIV infection. Such MK-mediated autocrine and paracrine mechanisms against HIV infection might be functional in infected individuals. 
Autocrine activity of MK: inhibition of HIV infection in cem T-cell clones expressing MK constitutively

CEM CD4+ T cells are human lymphoid cells permissive to infection by the X4 HIV-1 LAI isolate. These cells were used as parental cells to establish by stable transfection CEM clones expressing MK constitutively. The production of $\mathrm{MK}$ in the different clones was monitored by immunoblotting using crude cell extracts in order to select the positive clones. In parallel FACS analysis carried out on the selected clones for the expression of CD4 and CXCR4 revealed no apparent modification between the MK-producing clones and the corresponding control CEM clones. Consistent with the anti-HIV effect of the exogenously added MK, HIV-1 LAI infection was inhibited in the MKproducing clones compared to the parental CEM cells and the clone transfected with the plasmid alone. The degree of inhibition of HIV infection in MK producing cell lines was correlated with the level of recombinant MK expression. As for HIV particle attachment and entry, there was a strong inhibition in the MK producing clones whereas no effect occurred in the corresponding control clones. The results obtained with these MK producing clones indicate that HIV infection could be inhibited in cells by the endogenously produced MK, as we demonstrated to be the case with the exogenously added MK.

Paracrine activity of $M K$ : resistance of hiv permissive cells to infection by coculturing with MK producing cells.

Parental HeLa cells are not permissive to HIV infection due to the lack of CD4 expression. These cells were used to establish by transfection CD4- HeLa cell clones producing MK. Immunoblotting crude HeLa cell extracts with antiMK antibodies permitted the selection of several HeLa clones producing MK. The production of MK was also monitored by confocal immunofluorescence microscopy in the positive clones in which MK was localized in the nucleolus and abundantly at the cell surface. No significant amounts of MK were detectable in the culture supernatants of MK producing cells thus suggesting that secreted MK binds rapidly to its cell-surface receptor.

In order to demonstrate that MK produced by a given cell can protect a non-producer bystander cell against HIV infection, HIV permissive HeLa P4 cells were cocultured for $24 \mathrm{~h}$ with the CD4- HeLa cell clones producing MK before infection by the HIV-1 LAI isolate. At the effector (HeLa/MK cell clones) to target (HeLa P4 cells) ratio of 1 : 1 , the degree of net inhibition of HIV infection was $61-93 \%$ depending the respective MK producing HeLa clones; the degree of HIV-inhibition being correlated to the degree of recombinant $\mathrm{MK}$ expression. In further experiments, we then demonstrated that such MK-induced anti-HIV LAI resistance is also effective against other viral isolates, such as HIV-1 Ba-L and HIV-2 ROD. On the other hand, HIV-1 pseudotyped with MoMLV envelope glycoproteins was not affected consistent with the specific action of MK on HIV-envelope mediated viral infection.

Such coculturing experiments of HIV permissive with non-permissive cells suggest that MK produced by CD4cells can transmit protection of CD4+ bystander cells against HIV infection. Indeed, when CD4+ cells that are negative for the MK expression, are cocultured with CD4cells producing MK, they become positive for $\mathrm{MK}$ at their cell surface; thus illustrating the transfer of MK from one cell type to the other [3].

\section{The binding of MK to cells does not require heparan- and chondroitin-sulfate proteoglycans}

The binding of ${ }^{125}$ I-labeled MK to HIV permissive $\mathrm{HeLa} \mathrm{P} 4$ cells is in a dose-dependent manner. At $20^{\circ} \mathrm{C}$, the ${ }^{125}$ I-labeled MK is localized mostly on the cell surface since trypsin-treatment of cells eliminates more than $95 \%$ of bound MK. Furthermore, this binding is specific since it is reduced by excess unlabeled MK. Interestingly, the binding of the ${ }^{125}$ I-labeled MK to cells is significantly reduced by HIV-1 LAI, consistent with the suggestion that MK and HIV particles interact with common cell surface component(s).

In the literature, heparan- and chondroitin-sulfate proteoglycans have been reported to bind soluble preparations of MK [20]. In order to evaluate the potential requirement of heparan- and chondroitin-sulfate proteoglycans in the capacity of MK to bind the cell surface, we used Chinese hamster ovary mutant cell lines that are deficient in the expression of heparan sulfate (CHO 677) or both heparan/ chondroitin-sulfate proteoglycans (CHO 618) [21]. Despite lacking proteoglycan expression, these cell lines expressed the cell-surface nucleolin as revealed by the capacity of the biotin-labeled HB-19 to bind and form a stable complex with it [4]. All binding experiments were carried out at $20^{\circ} \mathrm{C}$ to prevent MK entry into cells. We first investigated the specific and non-specific binding of the ${ }^{125}$ I-labeled MK to the wild type CHO K1 cells that express heparan- and chondroitin-sulfate proteoglycans by washing cells at 300 and $150 \mathrm{mM} \mathrm{NaCl}$, respectively. In cells washed with 300 $\mathrm{mM} \mathrm{NaCl}$, specific binding occurs in a dose dependent manner and reaches a saturation at $1.2 \mu \mathrm{M}$ of $\mathrm{MK}$, at a dose that inhibits more than 95\% HIV infection in different types of cells [3]. A similar saturation value is obtained in $\mathrm{HeLa}$ P4 and Wilms' tumor cells. Interestingly, the ${ }^{125}$ I-labeled MK binding profile to heparan sulfate deficient $\mathrm{CHO} 677$ and to both heparan- and chondroitin-sulfate proteoglycan deficient CHO 618 cells is similar to that observed for the wild type $\mathrm{CHO} \mathrm{K} 1$ cells. These results therefore indicate 
that both heparan- and chondroitin-sulfate proteoglycans are not implicated in the mechanism of binding of MK under our experimental conditions [4]. Consistent with this, the binding of MK to cells is not affected by FGF-2 that uses heparan-sulfate proteoglycans as low affinity receptors [3].

Since high affinity binding sites for MK have been reported on some cell lines [9], we investigated the presence of such sites on $\mathrm{CHO}$ cells. Indeed, we found out that all the $\mathrm{CHO}$ cell lines express high affinity binding sites. Scatchard analysis of the ${ }^{125}$ I-labeled MK binding using high and low concentrations confirmed the presence of low affinity and high affinity binding sites, respectively [4]. The estimated $\mathrm{Kd}$ value for MK binding to the high affinity binding site on both $\mathrm{CHO} \mathrm{K} 1$ and $\mathrm{CHO} 618$ cells is $1.5 \mathrm{nM}$ with an estimated 110,000 sites per cell. The estimated Kd value for MK binding to the low affinity binding site on CHO K1 and CHO 618 cells is $3.6 \times 10^{-7}$ (900 000 sites/cell) and 1.8 x $10^{-7} \mathrm{M}$ (1 800000 sites/cell), respectively.

\section{The nucleolin-binding HB-19 pseudopeptide blocks the binding of MK to the low but not high affinity binding site [4]}

The different $\mathrm{CHO}$ cell lines were employed to investigate competition experiments for the low and high affinity binding sites. The specificity of the ${ }^{125} \mathrm{I}$-labeled MK binding to the low affinity binding site was confirmed by the fact that unlabeled MK prevented this binding. The nucleolin-binding HB-19 pseudopeptide [1, 14, 15] drastically inhibited the ${ }^{125}$ I-labeled MK binding to the low affinity site. On the other hand, the N-terminal tail peptide of MK or the C-terminal tail peptide of PTN had no apparent effect. The peptide corresponding to the C-terminal tail of PTN contains 11 lysine residues [8]. Consequently, the capacity of HB-19 to prevent the binding of MK to the low affinity binding site can not be accounted simply by the basic nature of HB-19 [2]. This latter was further confirmed by the use of the anti-HIV 9Arg peptide, which is composed of $9 \mathrm{D}$-arginine residues.

The binding of the ${ }^{125}$ I-labeled MK to the high affinity binding site was also specific as it was prevented by unlabeled MK. However, the HB-19 pseudopeptide had no effect on MK binding to the high affinity binding site, so as the 9Arg peptide and the MK and PTN derived peptides. These observations illustrate that the high affinity binding site is a distinct receptor different from the low affinity binding site. It is of interest to note that the concentration of MK necessary for the growth factor action of $\mathrm{MK}$ is around $5 \mathrm{nM}$ [8], whereas that for the anti-HIV action is $0.5 \mu \mathrm{M}$ [3]. Consequently, the growth factor and anti-HIV action of MK should be triggered independently through the interaction of MK with the high and low affinity binding site, respectively. The concentration required for the anti-HIV action of MK, and the fact that HB-19 prevents the binding of MK to the low affinity binding site, strongly suggest that this site is the cell-surface-expressed nucleolin.

The specificity of MK binding to the nucleolin band was investigated by ligand blotting experiments, in which we used ${ }^{125}$ I-labeled MK and purified preparations of nucleolin from HeLa P4 cells [4]. By this approach, we demonstrated that the binding of MK to the denatured nucleolin band is through a direct and specific interaction. The binding of MK to the nucleolin band was inhibited in a dose dependent manner by unlabeled excess MK and HB-19, whereas the control peptides had no effect.

\section{The tight interaction of MK with the cell-surface expressed nucleolin}

In order to confirm the interaction of the exogenously added MK to the cell surface expressed nucleolin, confocal microscopy studies were performed using a specific monoclonal antibody against human nucleolin and goat polyclonal antibody against MK [4]. We first demonstrated that MK bound specifically to cells (HeLa and $\mathrm{CHO}$ ) at $4^{\circ} \mathrm{C}$ is revealed by a signal, which is somewhat evenly distributed at the cell periphery, and this MK signal colocalizes with that of the surface nucleolin. Constantly, we observed that a small proportion of the specifically bound MK becomes tightly associated with the cell surface and resists $2 \mathrm{M}$ salt wash. In order to investigate whether under such drastic washing conditions MK is colocalized with nucleolin, HeLa P4 cells were incubated with $\mathrm{MK}$ at $37^{\circ} \mathrm{C}$ for $30 \mathrm{~min}$ before washing cells with culture medium containing $2 \mathrm{M} \mathrm{NaCl}$. By this procedure, only MK that has become anchored in the plasma membrane is detectable. After 7\% PFA fixation, cells were labeled using the antibodies against MK and nucleolin. The results show that MK is concentrated in distinct spots at the plasma membrane and it colocalizes with the nucleolin spots. The colocalization of the MK signal with that of surface nucleolin is in accord with the results observed in activated $\mathrm{T}$ lymphocytes in which the MK signal colocalizes with that of nucleolin.

Finally, the cross linking of surface bound MK with specific anti-MK antibodies, results in clustering of surface nucleolin and its colocalization with the MK signal. This is a specific event since under similar experimental conditions the distribution of the protein phosphatase CD45 is not significantly affected [3, 4]. This ligand-dependent clustering of a receptor is consistent with surface nucleolin being the main target of MK. 


\section{The C-terminal tail of nucleolin containing the RGG repeats is the site of binding to MK}

Three main structural domains have been determined in nucleolin: (1) the amino terminal domain containing several long stretches of acidic residues, (2) the central globular domain containing four RNA-binding domains (RBDs), and (3) the extreme C-terminal domain containing nine repeats of the tripeptide motif arginine-glycineglycine (RGG domain) [10, 11]. We generated truncated constructs of the C-terminal part of nucleolin in E. coli fused with GST as a tag to permit their purification using glutathione sepharose chromatography [17]. The expression of these constructs was monitored by immunoblotting using crude bacterial extracts and an anti-GST antibody. The MK binding capacity of each construct was then investigated by incubation of crude bacterial extracts with the ${ }^{125}$ I-labeled MK and the complexes were purified using glutathione sepharose chromatography. The results show that the presence of the RGG domain determines the MK binding capacity of a given construct in the $\mathrm{C}$-terminal part of nucleolin [4]. Furthermore, the RGG domain alone is sufficient for binding. Thus the RGG domain in nucleolin is the binding site for MK. Consistent with this, HB-19 that also binds the RGG domain [17] is a potent inhibitor of MK binding to nucleolin.

\section{Internalization of MK by an active process is inhib- ited by the nucleolin-binding HB-19 pseudopeptide and it requires the expression of LRP}

MK is internalized in different types of cells incubated at $37^{\circ} \mathrm{C}$. As heparan sulfate proteoglycans are implicated in the internalization of FGF-2 [22], we investigated the entry of MK in the heparan- and both heparan/chondroitin-deficient $\mathrm{CHO}$ cell lines [4]. The results indicate that MK entry is as efficient in the heparan-sulfate-deficient CHO 677 and heparan/Chondroitin-sulfate deficient CHO 618 cells as in the wild type CHO KI cells. This effect is specific to MK since FGF-2 entry is restricted only in the wild type CHO K1 cells. This latter is in accord with previous results showing that internalization of FGF-2 in $\mathrm{CHO}$ cells is dependent on the expression of heparan sulfate proteoglycans since $\mathrm{CHO}$ cells lack expression of high affinity receptors for FGF-2 [22].

Internalization of MK was further investigated in HeLa cells. Entry of MK was in a time- and dose-dependent manner, and reached saturation around $1 \mu \mathrm{M}$ concentration of MK. Internalization was significantly reduced when cells were incubated in the absence of FCS, whereas no entry occurred when cells were incubated at $20^{\circ} \mathrm{C}$ even in the presence of $10 \%$ FCS. These observations indicate that internalization of MK occurs by an active process. Sodium azide, an inhibitor of classical endocytosis via clathrin-coated vesicles, had no effect on MK internalization [4]. As it was expected, no MK entry was observed in the presence of HB-19 that inhibits the binding of MK to cells. Internalized MK remained blocked in the cytoplasm and even prolonged incubation did not result in its entry in the nucleus. However MK entered cell nuclei freely when cholesterol in the plasma membrane was eliminated by the drug methyl-beta-cyclodextrin (CD) [23]. MK entry in CDtreated cells is most probably by a passive process, since MK entry into cell nuclei also occurred in CD-treated cells incubated at $20^{\circ} \mathrm{C}$. Therefore, elimination of cholesterol leads to a receptor-independent entry of $\mathrm{MK}$, which is then accessible to the nuclei. However, in untreated cells, MK entry is by an active process in which membrane-cholesterol and lipid rafts appear to be implicated [4]. Indeed, the cross-linking of surface bound MK with a specific antibody results in the clustering of surface nucleolin along with glycosylphosphatidylinositol-linked proteins CD90 and CD59, pointing out that $\mathrm{MK}$ binding induces lateral assemblies of nucleolin with specific membrane components of lipid rafts. Accordingly, destruction of membrane cholesterol, which is an essential component for the formation of lipid rafts, results in MK internalization by a receptor-independent passive process [4].

The active entry of MK requires the presence of LRP, which is one of the high affinity receptors of MK. This latter is due to the lack of expression of surface nucleolin in mutant $\mathrm{CHO}$ cells that do not express LRP (unpublished data). Indeed, surface nucleolin is detectable in LRP + ve but not in LRP -ve cells, although in both cell types nucleolin is expressed abundantly in the cytoplasm and in the nucleus. Interestingly, there is a differential mechanism for MK entry in LRP + ve and LRP - ve cells. Whereas MK entry is blocked at $20^{\circ} \mathrm{C}$ in LRP +ve cells, MK entry is not affected in LRP -ve cells at $20^{\circ} \mathrm{C}$. These observations indicate that MK entry in LRP + ve cells is by an active process, whereas in LRP -ve cells it is by a passive process, most probably due to the absence of surface nucleolin. As nucleolin does not have a hydrophobic domain for its anchorage in the plasma membrane, LRP could be the nucleolin partner that allows surface expression of nucleolin and thus its proper functioning. This is in accord with the data of Shibata et al [24] who have reported that both LRP and nucleolin are necessary for MK endocytosis. However, in our experiment MK becomes concentrated in the cytoplasm and does not enter the nucleus, whereas in the experiments of Shibata et al, the internalized MK is translocated to the nucleus. The discrepancy between our results and those of Shibata et al might be the different cell systems used. 


\section{Potential role of MK as a cytokine during HIV in- fection}

Cytokines are important mediators of homeostasis. They are produced by a variety of cells and act via membrane receptors on responsive cells. The mRNA encoding the full length MK precursor protein is expressed in vivo in normal adult tissue, at least, in the PBLs of healthy individuals. Moreover, in vitro treatment of lymphocyte by various stimuli induces a marked increase of MK mRNA expression. Furthermore, MK becomes readily detectable at the surface of activated lymphocytes. In view of its various growth factor effects [7-9], its enhanced expression in response to physiological agents, along with its specific interaction with target cells, it is plausible to consider MK as a cytokine that has a potential function in homeostasis.

The fact that MK is induced by extracellular stimuli during events that also result in T cell activation is consistent with cytokine nature of MK. The cytokine family includes proteins such as interleukins, interferons, and chemokines that mediate signals between cells implicated in the immune system as well as other cell populations. The expression of cytokine genes is highly regulated as their transcription becomes initiated upon activation and then shuts down even in the continuous presence of stimulating agents. In accord with this, MK expression is induced markedly but transiently during $\mathrm{T}$ cell activation of PBLs by PHA or mAbs anti-CD3/anti-CD28. Treatment of PBLs with IL-2 or IFN- $\gamma$ also results in the induction of MK mRNA, thus suggesting that these cytokines are potential candidates responsible for the induction of MK during the $\mathrm{T}$ cell activation process. Of particular interest is MK induction in PBL treated with $\mathrm{mAb}$ anti-CD28 but not $\mathrm{mAb}$ anti-CD3 alone, since for the activation of $\mathrm{T}$ lymphocytes the combined signaling of the $\mathrm{T}$ cell receptor-CD3 complex and the costimulatory CD28 molecule is necessary. The MK induction following engagement of the $\mathrm{CD} 28$ receptor alone is most probably the consequence of IL-2 production. Indeed, the IL-2 enhancer contains a functional motif named CD28 response element that serves as a target for mitogenic $\mathrm{T}$ cell activation signals [25]. Furthermore, the increased IL-2 production is strictly dependent on CD28 costimulation and does not appear to occur with CD3 stimulation alone [26].

Cytokines through autocrine or paracrine way play a crucial role in HIV pathogenesis [27]. Indeed, cytokines can directly or indirectly affect HIV disease progression by modulating the immune system on the one hand and by regulating virus infection on the other hand. Here we demonstrated that MK-treated cells are protected against infection by both $\mathrm{T}$ lymphocyte- and macrophage-tropic $\mathrm{HIV}$ isolates. Our results also illustrate that MK producing CD4+ cells are protected against HIV infection while
MK producing CD4- cells can provide a protection to the non-producer $\mathrm{CD} 4+$ bystander cells. In view of the potent anti-HIV action of MK, its in vivo expression in blood lymphocytes and the marked in vitro induction in response to physiological stimuli, it is plausible to suggest that MK is a cytokine that could regulate HIV infection in an autocrine and paracrine manner and thus be implicated in the evolution of HIV disease. In this respect, the potential contribution of MK in HIV pathogenesis needs to be defined along with previously described cytokines modulating the outcome of HIV infection.

\section{Acknowledgements}

The members of the laboratory of Ara Hovanessian who contributed to this work are Christian Callebaut, Sebastien Nisole, Elias Said, Josette Svab, and Bernard Krust. The work was supported by grants from CNRS (Centre National de la Recherche Scientifique) et ANRS (Agence Nationale de la Recherche sur le SIDA), France.

\section{References}

1 Nisole S, Krust B, Callebaut C, et al. The anti-HIV pseudopeptide HB-19 forms a complex with the cell-surface expressed nucleolin independent of heparan sulfate proteoglycans. J Biol Chem 1999; 274:27875-84.

2 Nisole S, Krust B, Dam E, et al. The HB-19 pseudopeptide $5\left[\mathrm{Ky}\left(\mathrm{CH}_{2} \mathrm{~N}\right) \mathrm{PR}\right]-\mathrm{TASP}$ inhibits attachment of T-lymphocyte- and macrophage-tropic HIV to permissive cells., AIDS Res Hum Retroviruses 2000; 16:237-49.

3 Callebaut C, Nisole S, Briand JP, Krust B, Hovanessian AG. Inhibition of HIV infection by the cytokine midkine. Virology 2001; 281:248-64.

4 Said AE, Krust B, Nisole S, Briand JP, Hovanessian AG. The anti-HIV cytokine midkine binds the cell-surface-expressed nucleolin as a low affinity receptor, J Biol Chem 2002; 277:37492-502.

5 Said EA, Courty J, Svab J, et al. Pleiotrophin inhibits HIV infection by binding the cell surface expressed nucleolin. FEBS J 2005; 272:4646-59.

6 Legrand D, Vigie K, Said EA, et al. Surface nucleolin participates in both the binding and endocytosis of lactoferrin in target cells. Eur J Biochem 2004; 271:303-17.

7 Kurtz A, Schulte AM, Wellstein A. Pleiotrophin and midkine in normal development and tumor biology. Crit Rev Oncog 1995; 6:151-77.

8 Muramatsu T. The midkine family of growth differentiation factors. Dev Growth Differ 1994; 36:1-8.

9 Kadomatsu K, Muramatsu T. Midkine and pleiotrophin in neural development and cancer. Cancer Lett 2004; 204:127-43.

10 Ginisty H, Sicard H, Roger B, Bouvet P. Structure and functions of nucleolin. J Cell Science 1999; 112:761-72.

11 Srivastava M, Pollard, HB. Molecular dissection of nucleolin's role in growth and cell proliferation: new insights. FASEB J 1999; 13:1911-22. 
12 Hovanessian AG, Puvion-Dutilleul F, Nisole S, et al. The cell-surface-expressed nucleolin is associated with the actin cytoskeleton. Exp Cell Res 2000; 261:312-28.

13 Callebaut C, Jacotot E, Guichard G, et al. Inhibition of HIV infection by pseudopeptides blocking viral envelope glycoprotein-mediated membrane fusion and cell death. Virology 1996; 218:181-192.

14 Callebaut C, Jacotot E, Krust B, et al. Pseudopeptides TASP inhibitors of HIV infection block viral entry by binding to a 95 kDa cell surface protein. J Biol Chem 1997; 272:7159-66.

15 Callebaut C, Blanco J, Benkirane $\mathrm{N}$, et al. Identification of V3 loop-binding proteins as potential receptors implicated in the binding of HIV particles to $\mathrm{CD}^{+}$cells. J Biol Chem 1998; 273:21988-97.

16 Nisole S, Krust B, Hovanessian AG. Anchorage of HIV on permissive cells leads to co-aggregation of viral particles with surface nucleolin at membrane raft microdomains. Exp Cell Res 2002; 276:155-73.

17 Nisole S, Said EA, Mische C, et al. The anti-HIV pentameric pseudopeptide HB-19 binds the C-terminal end of nucleolin and prevents anchorage of virus particles in the plasma membrane of target cells. J Biol Chem 2002; 277:20877-86.

18 Christian S, Pilch J, Akerman ME, et al. Nucleolin expressed at the cell surface is a marker of endothelial cells in angiogenic blood vessels. J Cell Biol 2003; 163:871-8.
19 Ye J, Young HA. Negative regulation of cytokine gene transcription. FASEB J 1997; 11:825-33.

20 Zou K, Muramatsu H, Ikematsu S, et al. A heparin-binding growth factor, midkine, binds to chondroitin sulfate proteoglycan, PGM/versican. Eur J Biochem 2000; 267:4046-53.

21 Esko JD, Rostand KS, Weinke JL. Tumor formation dependent on proteoglycan biosynthesis. Science 1988; 241:1092-6.

22 Roghani M, Moscatelli D. Basic fibroblast growth factor is internalized through both receptor-mediated and heparan sulfatemediated mechanisms. J Biol Chem 1992; 267:22156-62.

23 Yancey PG, Rodrigueza WV, Kilsdonk EP, et al. Cellular cholesterol efflux mediated by cyclodextrins. Demonstration of kinetic pools and mechanism of efflux. J Biol Chem 1996; 271:16026-34.

24 Shibata Y, Muramatsu T, Hirai M, et al. Nuclear targeting by the growth factor midkine. Mol Cell Biol 2002; 22:6788-96.

25 Civil A, Bakker A, Rensink I, et al. Nuclear appearance of a factor that binds the $\mathrm{CD} 28$ response element within the interleukin-2 enhancer correlates with interleukin-2 production. J Biol Chem 1996; 271:8321-7.

26 Ott M, Emiliani S, Van Lint C, et al. Immune hyperactivation of HIV-1 infected T cells mediated by Tat and the CD28 pathway. Science 1997; 275:1481-5.

27 Pantaleo G, Graziosi C, Fauci AS. New concepts in the immunopathogenesis of human immunodeficiency virus infection. $\mathrm{N}$ Engl J Med 1993; 328:327-35. 